\title{
Locally Well-Dominated and Locally Independent Well-Dominated Graphs
}

\author{
Igor E. Zverovich ${ }^{* \dagger}$ \\ RUTCOR - Rutgers Center for Operations Research \\ Rutgers, The State University of New Jersey \\ Piscataway, NJ 08854-8003 \\ USA \\ Vadim E. Zverovich* \\ Department of Mathematical Sciences \\ Brunel University, Uxbridge \\ Middlesex, UB8 3PH \\ UK \\ Vadim.Zverovich@brunel.ac.uk
}

\begin{abstract}
In this article we present characterizations of locally well-dominated graphs and locally independent well-dominated graphs, and a sufficient condition for a graph to be $k$-locally independent well-dominated. Using these results we show that the irredundance number, the domination number and the independent domination number can be computed in polynomial time within several classes of graphs, e.g., the class of locally well-dominated graphs.
\end{abstract}

Keywords: locally well-dominated graphs; irredundance number; domination number; independent domination number 05C75, $05 \mathrm{C69}$

*Supported by the INTAS and the Belarus Government (Project INTAS-BELARUS 97-0093).

${ }^{\dagger}$ Supported by RUTCOR. 


\section{Introduction}

All graphs will be finite and undirected, without loops or multiple edges. If $G$ is a graph, $V(G)$ denotes the set, and $|G|$ the number, of vertices in $G$. Let $N(x)$ denote the neighborhood of a vertex $x$, and let $\langle X\rangle$ denote the subgraph of $G$ induced by $X \subseteq V(G)$. Also let $N(X)=\cup_{x \in X} N(x)$ and $N[X]=N(X) \cup X$. Denote by $\delta(G)$ the minimal degree of vertices in $G$.

A set $I \subseteq V(G)$ is called independent if no two vertices of $I$ are adjacent. A set $X$ is called a dominating set if $N[X]=V(G)$. We say that a vertex $v \in V(G)$ dominates a set $X \subseteq V(G)$ if $X \subseteq N[v]$. An independent dominating set is a vertex subset that is both independent and dominating, or equivalently, is maximal independent. The independence number $\alpha(G)$ is the maximum cardinality of a (maximal) independent set of $G$, and the independent domination number $i(G)$ is the minimum cardinality taken over all maximal independent sets of $G$. The domination number $\gamma(G)$ is the minimum cardinality of a dominating set of $G$, and the upper domination number $\Gamma(G)$ is the maximum cardinality taken over all minimal dominating sets of $G$.

For $X \subseteq V(G)$ and $x \in X$, the set

$$
P N(x, X)=P N_{G}(x, X)=N[x]-N[X-\{x\}]
$$

is called the private neighborhood of $x$. Note that $x \in P N(x, X)$ if and only if $x$ is an isolated vertex in $\langle X\rangle$. It is well-known that a dominating set $X$ is minimal if and only if $P N(x, X) \neq \emptyset$ for any vertex $x \in X$.

If $P N(x, X)=\emptyset$, then $x$ is said to be redundant in $X$. A set $X$ containing no redundant vertex is called irredundant. The irredundance number $\operatorname{ir}(G)$ is the minimum cardinality taken over all maximal irredundant sets of $G$, and the upper irredundance number $I R(G)$ is the maximum cardinality of a (maximal) irredundant set of $G$.

A graph $G$ is called domination perfect (upper domination perfect, irredundance perfect, upper irredundance perfect) if $\gamma(H)=i(H)(\Gamma(H)=\alpha(H)$, ir $(H)=\gamma(H)$, $I R(H)=\Gamma(H)$, respectively), for every induced subgraph $H$ of $G$. We will need several results on the above classes of graphs.

The known characterization of domination perfect graphs in terms of 17 forbidden induced subgraphs [20] implies the following sufficient condition for a graph to be domination perfect.

Theorem A If a graph $G$ does not contain any of the graphs $G_{2}, G_{4}, G_{6}, G_{8}, G_{10}$ in Figure 1 as induced subgraphs, then $G$ is domination perfect.

Favaron [7] conjectured that if a graph $G$ does not contain three induced subgraphs, then $G$ is irredundance perfect. This conjecture was proved by Volkmann and Zverovich [18] and independently by Puech [15]:

Theorem B If a graph $G$ does not contain any of the graphs $G_{3}, G_{4}, G_{6}$ in Figure 1 as induced subgraphs, then $G$ is irredundance perfect. 
Two vertex subsets $A, B$ of a graph $G$ independently match each other if $A \cap B=$ $\emptyset,|A|=|B|$, and all edges between $A$ and $B$ form a perfect matching in $\langle A \cup B\rangle$. A graph $G$ of order $2 k$ is called a $W$-graph if there is a partition $V(G)=A \cup B$ such that $A$ and $B$ independently match each other. Clearly, $|A|=|B|=k$. The sets $A$ and $B$ are called parts, and the graph $G$ is denoted by $G(A, B)$. A graph $G$ is called minimal upper domination imperfect if $\alpha(G)<\Gamma(G)$ and $\alpha(H)=\Gamma(H)$, for every proper induced subgraph $H$ of $G$. The properties of minimal upper domination imperfect graphs proved in [21] are stated in Theorem C.

Theorem C If $G$ is a minimal upper domination imperfect graph, then $G=G(A, B)$ is a connected $W$-graph of order $2 k, \delta(G) \geq 2$, and $\alpha(G)=k-1$.

The next result is due to Gutin and Zverovich [9].

Theorem D Any upper domination perfect graph is upper irredundance perfect.

A graph $G$ is called $k$-locally well-dominated if for every induced subgraph $H$ of $G$ there exists a set $S \subseteq V(H)$ such that $|S| \leq k$ and each minimal dominating set of $H$ containing $S$ is a minimum dominating set. A graph $G$ is called $k$-locally independent well-dominated if for every induced subgraph $H$ of $G$ there exists an independent set $S \subseteq V(H)$ such that $|S| \leq k$ and each (minimal) independent dominating set of $H$ containing $S$ is a minimum independent dominating set. The class of 1-locally (independent) well-dominated graphs will be referred to as locally (independent) well-dominated graphs.

An investigation of $k$-locally (independent) well-dominated graphs is of particular interest, since the (independent) domination number can be computed polynomially for such graphs. The corresponding polynomial algorithms follow directly from the above definitions. Other classes of graphs having a polynomial time algorithm for calculating the domination and independent domination numbers can be found in $[1,2,3,4,5,6,8,12,14,16,17]$.

In this paper we present characterizations of locally well-dominated graphs and locally independent well-dominated graphs, and a sufficient condition for a graph to be $k$-locally independent well-dominated. Using these results and the above theorems on domination perfect graphs and irredundance perfect graphs, we show that the irredundance number, the domination number and the independent domination number can be computed in polynomial time within several classes of graphs, e.g., the class of locally well-dominated graphs.

\section{Locally Well-Dominated Graphs}

The following theorem provides a characterization of locally well-dominated graphs.

Theorem 1 A graph $G$ is a locally well-dominated graph if and only if $G$ does not contain any graph $G_{1}, G_{2}, \ldots, G_{15}$ in Figure 1 as an induced subgraph. 
Proof: The necessity follows from the fact that the graphs $G_{1}, G_{2}, \ldots, G_{15}$ are not locally well-dominated. To prove the sufficiency, let $G$ be a minimum counterexample, i.e., $G$ does not contain any of $G_{1}, G_{2}, \ldots, G_{15}$ as an induced subgraph, $G$ is not locally well-dominated, and $G$ has a minimum order. Let $D$ be a minimum dominating set of $G,|D|=\gamma(G)$. Since $G$ is not locally well-dominated and $G$ has a minimum order, it follows that for any vertex $v \in V(G)$ there is a minimal dominating set $M$ such that $v \in M$ and $|M|>\gamma(G)$. If $D=\{d\}$, then $D$ is the unique minimal dominating set containing $d$, a contradiction. Hence $\gamma(G) \geq 2$.

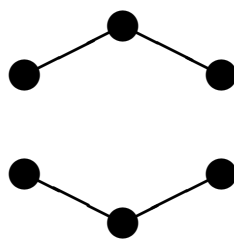

$G_{1}$

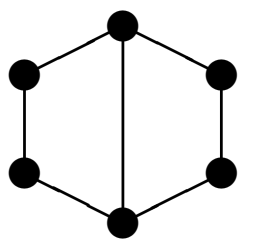

$G_{6}$

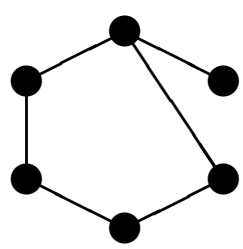

$G_{11}$

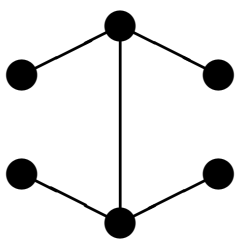

$G_{2}$

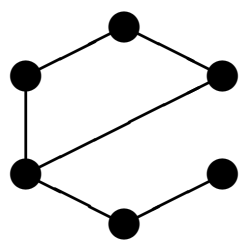

$G_{7}$

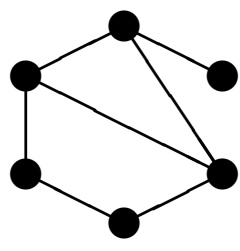

$G_{12}$

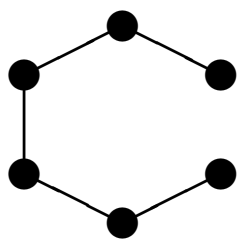

$G_{3}$

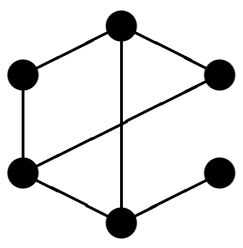

$G_{8}$

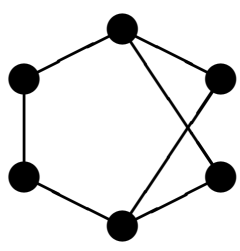

$G_{13}$

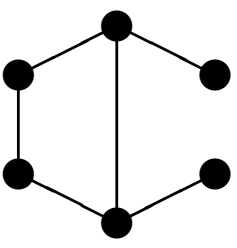

$G_{4}$

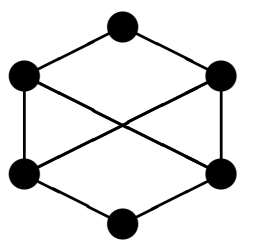

$G_{9}$

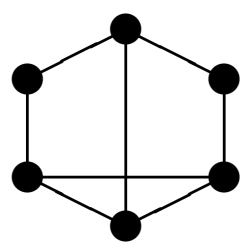

$G_{14}$

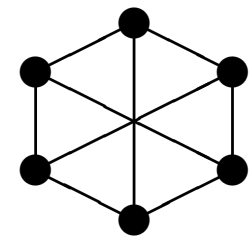

$G_{10}$

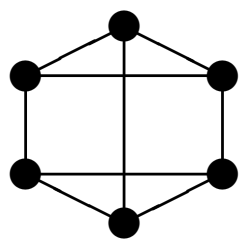

$G_{15}$

FIGURE 1. Graphs $G_{1}-G_{15}$.

Suppose that $\langle P N(d, D)\rangle$ is not a complete graph for any $d \in D$, and consider different vertices $c, d \in D, c^{\prime}, c^{\prime \prime} \in P N(c, D), d^{\prime}, d^{\prime \prime} \in P N(d, D)$ such that $c^{\prime} c^{\prime \prime} \notin$ $E(G)$ and $d^{\prime} d^{\prime \prime} \notin E(G)$. It is easy to see that $\left\langle\left\{c, c^{\prime}, c^{\prime \prime}, d, d^{\prime}, d^{\prime \prime}\right\}\right\rangle$ is isomorphic to one of the graphs $G_{1}, G_{2}, \ldots, G_{10}$, a contradiction. Therefore, there exists $x \in D$ such that $\langle P N(x, D)\rangle$ is a complete graph. Denote $X=P N(x, D) \cup\{x\}$ which induces a 
complete graph, and consider the graph $H=G-X$. Since $D-\{x\}$ is a dominating set of $H$, we have $\gamma(H)<\gamma(G)$.

We now show that an arbitrary vertex $v \in V(H)$ is contained in some minimal dominating set $M^{*}$ of $H$ such that $\left|M^{*}\right|>\gamma(H)$, thus proving the fact that $H$ is not locally well-dominated, contrary to the minimality of $G$. In the graph $G$ there is a minimal dominating set $M$ containing $v$ and such that $|M|>\gamma(G)$. We have $P N_{G}(u, M) \neq \emptyset$ for any vertex $u \in M$. There are three cases to consider.

Case 1: $|M \cap X| \geq 2$.

Denote $M^{\prime}=M-X$. Let us show that $X \cap P N_{G}(u, M)=\emptyset$ for any $u \in M$. Indeed, since $\langle X\rangle$ is a complete graph and $|M \cap X| \geq 2$, we see that $x \notin P N_{G}(x, M)$ for any $x \in M \cap X$. If $x \in X-M$, then $x$ is adjacent to at least two vertices of the set $M \cap X$, and hence $x$ cannot belong to any private neighborhood $P N_{G}(u, M)$. Thus, for any vertex $u \in M^{\prime}$,

$$
P N_{H}\left(u, M^{\prime}\right) \supseteq P N_{G}(u, M) .
$$

Moreover, the set $F=V(H)-N\left[M^{\prime}\right]$ is not empty, since it contains vertices of the sets $P N_{G}(x, M) \neq \emptyset$ for $x \in M \cap X$.

We now prove that an arbitrary vertex $f \in F$ does not dominate $P N_{H}\left(a, M^{\prime}\right)$ for any $a \in M^{\prime}$. Suppose to the contrary that $f$ dominates $P N_{H}\left(a, M^{\prime}\right)$. Since $f$ is not adjacent to $a$, we have $a \notin P N_{H}\left(a, M^{\prime}\right)$ and hence $a$ is adjacent to $b \in$ $M^{\prime}$. In the graph $G$, the vertex $f$ dominates $P N_{G}(a, M)$ and $f$ is adjacent to $x \in M \cap X$. Consider the graph $T=\left\langle\left\{a, a^{\prime}, b, b^{\prime}, x, f\right\}\right\rangle$, where $a^{\prime} \in P N_{G}(a, M)$ and $b^{\prime} \in P N_{G}(b, M)$. The only edges undetermined are $a^{\prime} b^{\prime}, b^{\prime} f, a x$ and $b x$. If none of them is present, then $T$ is isomorphic to $G_{3}$. If only one is present, then $T$ is isomorphic to $G_{7}$ or $G_{11}$. The presence of exactly two edges produces one of the graphs $G_{6}, G_{12}$ or $G_{13}$, while the presence of exactly three edges yields the graph $G_{14}$. At last, if all the above edges are present, then $T$ is isomorphic to $G_{15}$. Thus, the graph $T$ is isomorphic to one of the forbidden graphs $G_{3}, G_{6}, G_{7}, G_{11}, G_{12}, \ldots, G_{15}$, a contradiction. In fact, we have proved the following property.

Claim 1 Let $T$ be a graph with the vertex set $V(T)=\left\{a_{i}: i=1,2, \ldots, 6\right\}$ and such that $a_{i} a_{i+1} \in E(T)$ for $i=1,2, \ldots, 5$ and $a_{1} a_{3}, a_{1} a_{6}, a_{4} a_{2}, a_{4} a_{6}, a_{5} a_{2}, a_{5} a_{3} \notin E(T)$. Then $T$ is isomorphic to one of the graphs $G_{3}, G_{6}, G_{7}, G_{11}, G_{12}, \ldots, G_{15}$.

Let us show that the graph $\langle F\rangle$ contains a maximal independent set $J$ of cardinality at least $|M \cap X|-1$. If $|M \cap X|=2$, then we take an arbitrary maximal independent set $J$ of $F,|J| \geq 1$. Suppose that $|M \cap X| \geq 3$ and denote $P N_{x}=P N_{G}(x, M)$ for $x \in M \cap X$. We know that $P N_{x} \subset F$ for any $x \in M \cap X$. Moreover, there is no edge between $P N_{x}$ and $P N_{y}$ for any $x, y \in M \cap X$, for otherwise, taking three vertices of the set $M \cap X$ and three vertices from their private neighborhoods, we have one of the forbidden graphs $G_{12}, G_{14}$, or $G_{15}$. Now we can easily construct a maximal independent set $J$ of $\langle F\rangle$ of cardinality at least $|M \cap X|$ by including in $J$ one private neighbor of each vertex from $M \cap X$. 
The set $M^{*}=M^{\prime} \cup J$ is a dominating set of $H$ and minimal, since each vertex of $H$ has a nonempty private neighborhood. We have

$$
\left|M^{*}\right|=\left|M^{\prime} \cup J\right| \geq\left|M^{\prime}\right|+|M \cap X|-1=|M|-1 \geq \gamma(G)>\gamma(H) .
$$

Case 2: $|M \cap X|=1$.

Denote $M^{\prime}=M-X$. Since $\langle X\rangle$ is a complete graph, we have $X \cap P N_{G}(u, M)=\emptyset$ for any $u \in M^{\prime}$. Therefore, for any vertex $u \in M^{\prime}$,

$$
P N_{H}\left(u, M^{\prime}\right) \supseteq P N_{G}(u, M) .
$$

Denote $F=V(H)-N\left[M^{\prime}\right]$ and consider a maximal independent set $J$ of $\langle F\rangle$. We put $J=\emptyset$ if $F=\emptyset$. In the same way as in Case 1 we can prove that an arbitrary vertex $f \in F$ does not dominate $P N_{H}\left(a, M^{\prime}\right)$ for any $a \in M^{\prime}$. Thus, the set $M^{*}=M^{\prime} \cup J$ is a minimal dominating set of $H$ and

$$
\left|M^{*}\right|=\left|M^{\prime} \cup J\right| \geq\left|M^{\prime}\right|=|M|-1 \geq \gamma(G)>\gamma(H) .
$$

Case 3: $M \cap X=\emptyset$.

If $X$ contains no private neighborhood of a vertex from $M$, then $M$ is a minimal dominating set of $H$ and $|M|>\gamma(G)>\gamma(H)$. We therefore can assume that $X$ contains $k \geq 1$ private neighborhoods $P N_{G}\left(a_{i}, M\right), i=1,2, \ldots, k$. Since $a_{i} \notin$ $P N_{G}\left(a_{i}, M\right)$, we conclude that $a_{i}$ is not an isolated vertex in $\langle M\rangle$ for $i=1,2, \ldots, k$.

Suppose that $k \geq 3$ and consider the graph $\left\langle\left\{a_{1}, a_{2}, a_{3}, p_{1}, p_{2}, p_{3}\right\}\right\rangle$, where $p_{i} \in$ $P N_{G}\left(a_{i}, M\right), 1 \leq i \leq 3$. This graph is isomorphic to $G_{12}, G_{14}$, or $G_{15}$, unless the set $\left\{a_{1}, a_{2}, a_{3}\right\}$ is independent. In the case $\left\{a_{1}, a_{2}, a_{3}\right\}$ is independent, there exist the vertex $a \in M$ adjacent to $a_{1}$ and the vertex $p \in P N_{G}(a, M)$. By Claim 1, the graph $\left\langle\left\{p, a, a_{1}, p_{1}, p_{2}, a_{2}\right\}\right\rangle$ is isomorphic to one of the graphs $G_{3}, G_{6}, G_{7}, G_{11}, G_{12}, \ldots G_{15}$, a contradiction.

Consider the case $k=2$ and suppose that at least one of the vertices $a_{1}, a_{2}$, say the vertex $a_{1}$, is adjacent to $a \in M-\left\{a_{1}, a_{2}\right\}$. Now, taking the vertex $p \in$ $P N_{G}(a, M)$ and applying Claim 1 to the graph $\left\langle\left\{p, a, a_{1}, p_{1}, p_{2}, a_{2}\right\}\right\rangle$, we have one of the forbidden graphs, a contradiction. Therefore, $a_{1} a_{2}$ is an isolated edge in $\langle M\rangle$. We put $M^{*}=M-\left\{a_{1}\right\}$ if $v \neq a_{1}$, and $M^{*}=M-\left\{a_{2}\right\}$ if $v=a_{1}$. It is not difficult to see that every vertex of $M^{*}$ has a nonempty private neighborhood in $H$ and $M^{*}$ dominates $H$. Hence $M^{*}$ is a minimal dominating set of $H, M^{*}$ contains the vertex $v$, and

$$
\left|M^{*}\right|=|M|-1 \geq \gamma(G)>\gamma(H) .
$$

At last, suppose that $k=1$. If $v \neq a_{1}$, then $M^{*}=M-\left\{a_{1}\right\}$ is a minimal dominating set of $H$ and $\left|M^{*}\right|>\gamma(H)$. Assume that $v=a_{1}$ and construct the set $M^{\prime}=M-B$, where $B=N\left(a_{1}\right) \cap M \neq \emptyset$. Using the same argument as in Case 1 we see that an arbitrary vertex $f \in V(H)-N\left[M^{\prime}\right]$ does not dominate $P N_{H}\left(a, M^{\prime}\right)$ for any $a \in M^{\prime}$. Moreover, there is no edge between $P N_{G}\left(b_{1}, M\right)$ and $P N_{G}\left(b_{2}, M\right)$ for any $b_{1}, b_{2} \in B$, for otherwise there is a graph satisfying the conditions of Claim 1 
which produces a contradiction. Now we can easily construct a maximal independent set $J$ of $\langle F\rangle$ of cardinality $|J| \geq|B|$. The set $M^{*}=M^{\prime} \cup J$ is a minimal dominating set of $H$ and

$$
\left|M^{*}\right|=\left|M^{\prime} \cup J\right| \geq\left|M^{\prime}\right|+|B|=|M|>\gamma(G)>\gamma(H) .
$$

The proof of Theorem 1 is complete.

The next corollary follows directly from Theorem 1 and Theorems A and B.

Corollary 1 Any locally well-dominated graph is domination perfect and irredundance perfect.

The definition of locally well-dominated graphs easily implies a polynomial time algorithm for calculating the domination number for such graphs. By Corollary 1, the same algorithm yields the independent domination number and the irredundance number. It may be pointed out that the corresponding decision problems IRREDUNDANT SET and DOMINATING SET are both NP-complete on the class of irredundance perfect graphs [19], and the problems DOMINATING SET and INDEPENDENT DOMINATING SET are both NP-complete on domination perfect graphs [20].

Corollary 2 The parameters $\operatorname{ir}(G), \gamma(G)$ and $i(G)$ for locally well-dominated graphs can be computed in time $O\left(|G|^{3}\right)$.

Jacobson and Peters [10] showed that a number of known classes of graphs are both upper domination and upper irredundance perfect, and noted that it is interesting to find other classes having this property.

Corollary 3 Any locally well-dominated graph is upper domination perfect and upper irredundance perfect.

Proof: Suppose that the first statement is false and consider a minimal graph $G$ which is locally well-dominated and upper domination imperfect. It is evident that $G$ is a minimal upper domination imperfect graph. By Theorem C, $G=G(A, B)$ is a connected W-graph of order $2 k, \delta(G) \geq 2$, and $\alpha(G)=k-1$. By Theorem 1, $G$ does not contain any graph $G_{1}, G_{2}, \ldots, G_{15}$ in Figure 1 as an induced subgraph. Since $\delta(G) \geq 2$, the part $A$ contains an edge $a_{1} a_{2}$. Let $a_{1} b_{1}$ and $a_{2} b_{2}$ be edges of the perfect matching between the parts $A$ and $B$.

Assume that one of the vertices $b_{1}$ and $b_{2}$, say $b_{2}$, is adjacent to a vertex $b_{3} \in$ $B-\left\{b_{1}, b_{2}\right\}$, and let $b_{3} a_{3}$ be the corresponding edge of the perfect matching. Applying Claim 1 to the set $\left\{a_{i}, b_{i}: i=1,2,3\right\}$, we see that the graph $\left\langle\left\{a_{i}, b_{i}: i=1,2,3\right\}\right\rangle$ is isomorphic to one of the graphs $G_{3}, G_{6}, G_{7}, G_{11}, G_{12}, \ldots, G_{15}$, a contradiction. Hence both $b_{1}$ and $b_{2}$ are not adjacent to any vertex of $B-\left\{b_{1}, b_{2}\right\}$. Taking into account that $\delta(G) \geq 2$, we obtain $b_{1} b_{2} \in E(G)$. Thus, $b_{1} b_{2}$ is an isolated edge in $\langle B\rangle$. 
We know that $\alpha(G)=k-1$. Further, $k \geq 3$, for otherwise $\alpha(G)=k$, a contradiction. Since $G$ is a connected graph, one of the vertices $a_{1}$ and $a_{2}$, say $a_{2}$, is adjacent to a vertex $a_{3} \in A-\left\{a_{1}, a_{2}\right\}$, and let $a_{3} b_{3}$ be the corresponding edge of the perfect matching. The only edge undetermined in the graph $\left\langle\left\{a_{i}, b_{i}: i=1,2,3\right\}\right\rangle$ is $a_{1} a_{3}$. Now, if $a_{1}$ is not adjacent to $a_{3}$, then $\left\langle\left\{a_{i}, b_{i}: i=1,2,3\right\}\right\rangle$ is isomorphic to $G_{7}$, while if $a_{1}$ is adjacent to $a_{3}$, then $\left\langle\left\{a_{i}, b_{i}: i=1,2,3\right\}\right\rangle$ is isomorphic to $G_{12}$, a contradiction. Thus, any locally well-dominated graph is upper domination perfect and, by Theorem D, upper irredundance perfect.

We believe that the independence number (equal to both the upper domination number and the upper irredundance number) for locally well-dominated graphs can be computed in polynomial time. Other problems worth investigating are presented below.

Conjecture 1 The class of $k$-locally well-dominated graphs for any $k$ can be characterized in terms of a finite number of forbidden induced subgraphs.

Problem 1 Characterize the class of 2-locally well-dominated graphs.

\section{$3 \quad k$-Locally Independent Well-Dominated Graphs}

We say that a graph $G$ belongs to the family $\mathcal{M}_{k}$ if $G$ can be obtained by the following procedure. Let $H$ be a graph of order $2 k$ having a perfect matching. We subdivide each edge of the perfect matching $P$ by a single vertex, i.e., each edge $x_{i} z_{i}$ of $P$ is replaced by the chain $\left(x_{i}, y_{i}, z_{i}\right)$. The resulting graph $G$ belongs to $\mathcal{M}_{k}$. The next theorem provides a sufficient condition for a graph to be $k$-locally independent well-dominated in terms of the above family.

Theorem 2 If a graph $G$ does not contain any member of $\mathcal{M}_{k+1}$ as an induced subgraph, then $G$ is a $k$-locally independent well-dominated graph.

Proof: Let $G$ be a minimum counterexample, i.e., $G$ does not contain any member of $\mathcal{M}_{k+1}$ as an induced subgraph, $G$ is not a $k$-locally independent well-dominated graph, and $G$ has a minimum order.

Let $D$ be a minimum independent dominating set of $G,|D|=i(G)$. Since $G$ is not locally independent well-dominated and $G$ has a minimum order, it follows that for any independent set $I \subseteq V(G),|I| \leq k$, there is an independent dominating set $M$ such that $I \subseteq M$ and $|M|>i(G)$. We have $i(G) \geq k+1$, for otherwise $|D|=i(G) \leq k$ and $D$ is not contained in a larger independent dominating set. Consider the vertices $y_{1}, y_{2}, \ldots, y_{k+1} \in D$ and their private neighborhoods $P N\left(y_{i}, D\right)$, $i=1,2, \ldots, k+1$. Note that $y_{i} \in P N\left(y_{i}, D\right)$ for all $i=1,2, \ldots, k+1$. Suppose for every $i=1,2, \ldots, k+1$ that $\left\langle P N\left(y_{i}, D\right)\right\rangle$ is not a complete graph. Taking the vertices $y_{i}$ and choosing two nonadjacent vertices $x_{i}, z_{i} \in P N\left(y_{i}, D\right)-\left\{y_{i}\right\}$ for every 
$i=1,2, \ldots, k+1$, we see that the graph $\left\langle\left\{x_{i}, y_{i}, z_{i}: 1 \leq i \leq k+1\right\}\right\rangle$ belongs to the family $\mathcal{M}_{k+1}$, contrary to the hypothesis. Therefore, without loss of generality, $P N\left(y_{1}, D\right)$ induces a complete graph. Denote $X=P N\left(y_{1}, D\right)$ and consider the graph $H=G-X$. Since $D-\left\{y_{1}\right\}$ is an independent dominating set of $H$, we have $i(H)<i(G)$.

Let us show that an arbitrary independent set $I \subseteq V(H)$ of cardinality at most $k$ is contained in some independent dominating set $M^{*}$ of $H$ such that $\left|M^{*}\right|>i(H)$, thus proving the fact that $H$ is not locally independent well-dominated, contrary to the minimality of $G$. In the graph $G$ there is an independent dominating set $M$ containing $I$ and such that $|M|>i(G)$.

If $M \cap X=\emptyset$, then $M$ is an independent dominating set of $H$ and $|M|>i(G)>$ $i(H)$. If $M \cap X \neq \emptyset$, then $|M \cap X|=1$, since $\langle X\rangle$ is a complete graph. Denote $M \cap X=\{x\}$ and let $J$ be a maximal independent set of $F=\left\langle P N_{G}(x, M)-X\right\rangle$. We put $J=\emptyset$ if $F=\emptyset$. It is not difficult to see that the set $M^{*}=(M-\{x\}) \cup J$ is an independent dominating set of $H$ and

$$
\left|M^{*}\right|=|(M-\{x\}) \cup J| \geq|M-\{x\}|=|M|-1 \geq i(G)>i(H) .
$$

The proof is complete.

For the case $k=1$, Theorem 2 gives both sufficient and necessary conditions for a graph to be locally independent well-dominated. This follows from the fact that any graph of $\mathcal{M}_{2}$ is not locally independent well-dominated.

Corollary 4 A graph $G$ is a locally independent well-dominated graph if and only if $G$ does not contain any graph $G_{1}, G_{3}, G_{5}, G_{6}, G_{7}, G_{9}$ in Figure 1 as an induced subgraph.

The definition of $k$-locally independent well-dominated graphs and Theorem 2 imply a polynomial time algorithm for calculating the independent domination number within the class $\operatorname{FIS}\left(\mathcal{M}_{k}\right), k \geq 2$, i.e., the class of graphs having no induced subgraph from $\mathcal{M}_{k}$.

Corollary 5 For a fixed $k \geq 2$, the independent domination number within the class FIS $\left(\mathcal{M}_{k}\right)$ can be computed in time $O\left(|G|^{k+1}\right)$. In particular, for $k=2$, the independent domination number within the class $F I S\left(G_{1}, G_{3}, G_{5}, G_{6}, G_{7}, G_{9}\right)$ can be computed in time $O\left(|G|^{3}\right)$.

Note that the decision problems DOMINATING SET and IRREDUNDANT SET are both NP-complete on the class $\operatorname{FIS}\left(\mathcal{M}_{k}\right)$ for any $k \geq 2$, since this class generalizes split graphs for which the problems are known to be NP-complete [11, 13].

Our last result is proved with the use of Theorem 2 and Theorems A and B.

Corollary 6 The parameters $\operatorname{ir}(G), \gamma(G)$ and $i(G)$ within the class $F I S\left(G_{1}, \ldots, G_{4}\right.$, $\left.G_{6}, \ldots, G_{10}\right)$ can be computed in time $O\left(|G|^{4}\right)$. 
Proof: We firstly show that if a graph $G$ does not contain any graph $G_{1}, \ldots, G_{4}$, $G_{6}, \ldots, G_{10}$ as an induced subgraph, then $G$ is a 2-locally independent well-dominated graph. Suppose that $G$ contains an induced subgraph $H$ from $\mathcal{M}_{3}$. Let $\left(x_{i}, y_{i}, z_{i}\right)$, $i=1,2,3$, be the chains as in the definition of the family $\mathcal{M}_{k}$. Since the graphs $G_{1}, G_{3}, G_{6}, G_{7}, G_{9}$ are forbidden as induced subgraphs, it follows that the graph $\left\langle\left\{x_{i}, y_{i}, z_{i}, x_{j}, y_{j}, z_{j}\right\}\right\rangle$ is isomorphic to $C_{6}$ for any $i, j \in\{1,2,3\}, i \neq j$. Without loss of generality, we may assume that $x_{1} x_{2}, z_{1} z_{2}, x_{2} x_{3}, z_{2} z_{3} \in E(H)$. There are two cases to consider. If $x_{1} z_{3}, z_{1} x_{3} \in E(H)$, then $\left\langle\left\{x_{1}, x_{2}, x_{3}, z_{1}, y_{2}, y_{3}\right\}\right\rangle$ is isomorphic to $G_{2}$, a contradiction. If $x_{1} x_{3}, z_{1} z_{3} \in E(H)$, then $\left\langle\left\{y_{1}, z_{1}, z_{2}, y_{2}, x_{2}, x_{3}\right\}\right\rangle$ is isomorphic to $G_{3}$, a contradiction. Therefore, by Theorem 2, the graph $G$ is 2-locally independent well-dominated, and the necessary algorithm for the IDS problem easily follows. By Theorems A and B, $\operatorname{ir}(G)=\gamma(G)=i(G)$. The proof is complete.

We complete this section with the following problems.

Conjecture 2 The class of $k$-locally independent well-dominated graphs for any $k$ can be characterized in terms of a finite number of forbidden induced subgraphs.

Problem 2 Characterize the class of 2-locally independent well-dominated graphs.

Acknowledgment The authors thank the referees for valuable suggestions.

\section{References}

[1] Beyer, T.A., Proskurowski, A., Hedetniemi, S.T., Mitchell, S.: Independent domination in trees. Congr. Numer. 19, 321-328 (1977).

[2] Brandstädt, A., Kratsch, D.: On domination problems for permutation and other graphs. Theoretical Computer Sci. 54, 181-198 (1987).

[3] Cockayne, E.J., Goodman, S.E., Hedetniemi, S.T.: A linear algorithm for the domination number of a tree. Inform. Process. Lett. 4, 41-44 (1975).

[4] Elmallah, E.S., Stewart, L.K.: Domination in polygon graphs. Congr. Numer. 77, 63-76 (1990).

[5] Farber, M.: Independent domination in chordal graphs. Oper. Res. Lett. 1, 134-138 (1982).

[6] Farber, M., Keil, J.M.: Domination in permutation graphs. J. Algorithms 6, (1985) 309-321. 
[7] Favaron, O.: Stability, domination and irredundance in a graph. J. Graph Theory 10, 429-438 (1986).

[8] Finbow, A., Hartnell, B., Nowakowski, R.: Well-dominated graphs: A collection of well-covered ones. Ars Comb. 25A, 5-10 (1988).

[9] Gutin, G., Zverovich, V.E.: Upper domination and upper irredundance perfect graphs. Discrete Math. 190, 95-105 (1998).

[10] Jacobson, M.S., Peters, K.: A note on graphs which have upper irredundance equal to independence. Discrete Appl. Math. 44, 91-97 (1993).

[11] Johnson, D.S: The NP-completeness column: an ongoing guide. J. Algorithms 5, 147-160 (1984).

[12] Kratsch, D., Stewart, L.: Domination on cocomparability graphs. SIAM J. Discrete Math. 6 (3), 400-417 (1993).

[13] Laskar, R., Pfaff, J.: Domination and irredundance in split graphs. Tech. Report 430, Dept. Mathematical Sciences, Clemson Univ., 1983.

[14] Prisner, E., Topp, J., Vestergaard P.D.: Well covered simplicial, chordal and circular arc graphs. J. Graph Theory 21 (2), 113-119 (1996).

[15] Puech, J.: Irredundance perfection and $P_{6}$-free graphs. J. Graph Theory 29, (1998) 239-255.

[16] Ramalingamg, G., Pandu Rangan, C.: Total domination in interval graphs revisited. Inform. Process. Lett. 27, 17-21 (1988).

[17] Topp, J., Vestergaard, P.D.: Well irredundant graphs. Discrete Appl. Math. 63, 267-276 (1995).

[18] Volkmann, L., Zverovich, V.E.: A proof of Favaron's conjecture and a disproof of Henning's conjecture on irredundance perfect graphs. The 5th Twente Workshop on Graphs and Combin. Optimization, Enschede, May 1997, 215-217.

[19] Volkmann, L., Zverovich, V.E.: A disproof of Henning's conjecture on irredundance perfect graphs. Discrete Math. (to appear) (2001).

[20] Zverovich, I.E., Zverovich, V.E.: An induced subgraph characterization of domination perfect graphs. J. Graph Theory 20, 375-395 (1995).

[21] Zverovich, I.E., Zverovich, V.E.: A semi-induced subgraph characterization of upper domination perfect graphs. J. Graph Theory 31, 29-49 (1999). 